\title{
Endorsement of the 66/68 Joint Count for the Measurement of Musculoskeletal Disease Activity: OMERACT 2018 Psoriatic Arthritis Workshop Report
}

\author{
Alí Duarte-García (iD, Ying Ying Leung (iD), Laura C. Coates, Dorcas Beaton, Robin Christensen, \\ Ethan T. Craig, Maarten de Wit (D), Lihi Eder, Lara Fallon, Oliver FitzGerald, \\ Dafna D. Gladman (D), Niti Goel (iD), Richard Holland, Chris Lindsay, Lara Maxwell, \\ Philip Mease, Ana Maria Orbai (D), Beverley Shea, Vibeke Strand, Douglas J. Veale, \\ William Tillett, and Alexis Ogdie (i)
}

\begin{abstract}
Objective. The Psoriatic Arthritis (PsA) Core Domain Set for randomized controlled trials and longitudinal observational studies has recently been updated. The joint counts are central to the measurement of the peripheral arthritis component of the musculoskeletal (MSK) disease activity domain. We report the Outcome Measures in Rheumatology (OMERACT) 2018 meeting's approaches to seek endorsement of the 66/68 swollen and tender joint count (SJC66/TJC68) for inclusion in the PsA Core Outcome Measurement Set (COS).

Methods. Using the OMERACT Filter 2.1 Instrument Selection Process, the SJC66/TJC68 was assessed for (1) domain match, (2) feasibility, (3) numerical sense (construct validity), and (4) discrimination (test retest reliability, longitudinal construct validity, sensitivity in clinical trials, and thresholds of meaning). A protocol was designed to assess the measurement properties of the SJC66/TJC68 joint count. The results were summarized in a Summary of Measurement Properties table developed by OMERACT. OMERACT members discussed and voted on whether the strength of the evidence supported that the SJC66/TJC68 had passed the OMERACT Filter as an outcome measurement instrument for the PsA COS.

Results. OMERACT delegates endorsed the use of the SJC66/TJC68 for the measurement of the peripheral arthritis component of the MSK disease activity domain. Among patient research partners, $100 \%$ voted for a "green" endorsement, whereas among the group of other stakeholders, $88 \%$ voted for a "green" endorsement.

Conclusion. The SJC66/TJC68 is the first fully endorsed outcome measurement instrument using the OMERACT Filter 2.1 and the first instrument fully endorsed within the PsA COS. (First Release June 1 2019; J Rheumatol 2019;46:996-1005; doi:10.3899/jrheum.181089)
\end{abstract}

Key Indexing Terms:

OMERACT PSORIATIC ARTHRITIS OUTCOMES MEASURES CORE SET

From the Division of Rheumatology, Department of Medicine, and the Robert D. and Patricia E. Kern Center for the Science of Health Care Delivery, Mayo Clinic, Rochester, Minnesota; Division of Rheumatology, Department of Medicine, University of Pennsylvania, Philadelphia, Pennsylvania; Division of Rheumatology, Duke University School of Medicine, Durham, North Carolina; Kezar Life Sciences, South San Francisco; Amgen Inc., Thousand Oaks, California; Swedish-ProvidenceSt. John's Health Systems and University of Washington, Seattle, Washington; Division of Rheumatology, Johns Hopkins University School of Medicine, Baltimore, Maryland, USA; Department of Rheumatology and Immunology, Singapore General Hospital, Singapore, Singapore; University of Leeds, Leeds; University of Oxford, Oxford; Royal National Hospital for Rheumatic Diseases; University of Bath, Bath, UK; Musculoskeletal Health and Outcomes Research, St. Michael's Hospital; Institute for Work and Health; Department of Occupational Science and Occupational Therapy, Rehabilitation Sciences Institute and the Institute for Health Policy Management and Evaluation, University of Toronto; Department of Medicine, University of Toronto, Women's College Hospital; Department of Medicine, University of Toronto, Toronto Western Hospital, Toronto; Cochrane Musculoskeletal Group, Ottawa Hospital Research Institute, Centre for Practice-Changing Research; School of Epidemiology and Public Health, University of Ottawa, Ottawa, Ontario;
Pfizer Inc., Montreal, Quebec, Canada; Musculoskeletal Statistics Unit The Parker Institute, Bispebjerg and Frederiksberg Hospital, Copenhagen; Department of Rheumatology, Odense University Hospital, Odense, Denmark; Department of Medical Humanities, Patient Research Partner, Amsterdam University Medical Centre, Amsterdam, the Netherlands; Department of Rheumatology, St. Vincent's University Hospital; Conway Institute for Biomolecular Research, University College Dublin, Ireland; Royal Prince Alfred Hospital Medical Centre, Sydney, Australia.

Several parts of this study were funded by the Rheumatology Research Foundation Innovative Research Award (PI Ogdie) and some by R01-AR072363 (PI Ogdie). The Parker Institute, Bispebjerg and Frederiksberg Hospital (RC) is supported by a core grant from the Oak Foundation (OCAY-13-309). A.M. Orbai is a Jerome L. Greene Foundation Scholar and is supported in part by a research grant from the US National Institute of Arthritis and Musculoskeletal and Skin Diseases of the National Institutes of Health under award number P30-AR070254 (Core B), a Rheumatology Research Foundation Scientist Development award, and a Staurulakis Family Discovery award. A. Duarte-García is supported by the Mayo Clinic Robert D. and Patricia E. Kern Center for the Science of Health Care Delivery, which receives no industry funding.

A. Duarte-García, MD, Division of Rheumatology, Department of 
Psoriatic arthritis (PsA) is a chronic inflammatory musculoskeletal and skin disease that is clinically heterogeneous with distinct manifestations including peripheral arthritis, spondylitis, enthesitis, and dactylitis, as well as skin and nail features. Additionally, the disease affects many domains of patients' lives including fatigue, participation, and emotional well-being. The Group for Research and Assessment of Psoriasis and Psoriatic Arthritis (GRAPPA)-Outcome Measures in Rheumatology (OMERACT) working group developed a core domain set (Figure 1) to specify which key domains should be measured in randomized controlled trials (RCT) and longitudinal observational studies (LOS) for PsA. This was endorsed at the 2016 OMERACT meeting ${ }^{1,2}$. Since that time, many work streams have been initiated as part of the Core Outcome Measures for Psoriatic Clinical Trials (COMPACT) study ${ }^{3,4}$. The GRAPPA-OMERACT working group has been evaluating the measurement properties of multiple outcome measurement instruments to develop a PsA Core Outcome Measurement Set (COS) that would assist in standardizing what is measured in RCT and how they are measured (domains and instruments) ${ }^{5,6}$.

Medicine, Mayo Clinic, and Robert D. and Patricia E. Kern Center for the Science of Health Care Delivery, Mayo Clinic; Y.Y. Leung, MD, MBChB, Department of Rheumatology and Immunology, Singapore General Hospital; L.C. Coates, PhD, MBChB, University of Leeds, and University of Oxford; D. Beaton, PhD, MSc, BScOT, Musculoskeletal Health and Outcomes Research, St. Michael's Hospital, and Institute for Work and Health, and Department of Occupational Science and Occupational Therapy, Rehabilitation Sciences Institute and the Institute for Health Policy Management and Evaluation, University of Toronto;

R. Christensen, PhD, MSc, Musculoskeletal Statistics Unit: The Parker Institute, Bispebjerg and Frederiksberg Hospital, and Department of Rheumatology, Odense University Hospital; E.T. Craig, MD, MPH, Division of Rheumatology, Department of Medicine, University of Pennsylvania; M. de Wit, PhD, Department of Medical Humanities, Patient Research Partner, Amsterdam University Medical Centre; L. Eder, $M D, P h D$, Department of Medicine, University of Toronto, Women's College Hospital; L. Fallon, PhD, Pfizer Inc.; O. FitzGerald, MD, MBChB, Department of Rheumatology, St. Vincent's University Hospital, and Conway Institute for Biomolecular Research, University College Dublin; D.D. Gladman, MD, Department of Medicine, University of Toronto, Toronto Western Hospital; N. Goel, MD, Patient Research Partner, Division of Rheumatology, Duke University School of Medicine, and Kezar Life Sciences; R. Holland, MBChB, Royal Prince Alfred Hospital Medical Centre; C. Lindsay, PharmD, Patient Research Partner, Amgen Inc.; L. Maxwell, PhD, Cochrane Musculoskeletal Group, Ottawa Hospital Research Institute, Centre for Practice-Changing Research; P. Mease, MD, Swedish-Providence-St. John's Health Systems and University of Washington; A.M. Orbai, MD, MHS, Division of Rheumatology, Johns Hopkins University School of Medicine; B. Shea, PhD, Ottawa Hospital Research Institute, and School of Epidemiology and Public Health, University of Ottawa; V. Strand, MD, Biopharmaceutical Consultant; D.J. Veale, MD, St. Vincent's University Hospital, and University College Dublin; W. Tillett, PhD, MBChB, Royal National Hospital for Rheumatic Diseases, and the University of Bath; A. Ogdie, MD, MSCE, Division of Rheumatology, Department of Medicine,

University of Pennsylvania. Dr. Duarte-García and Dr. Leung are co-first authors. W. Tillett and Dr. Ogdie are co-senior authors.

Address correspondence to Dr. A. Ogdie, University of Pennsylvania, White Building Room 5023, 3400 Spruce St., Philadelphia, Pennsylvania 19104, USA.E-mail: alexis.ogdie@uphs.upenn.edu

Accepted for publication January 30, 2019
Among the domains included in the COS, musculoskeletal (MSK) disease activity is considered one of the most important for both patients and clinicians ${ }^{1}$. The MSK disease activity domain includes peripheral joints, enthesitis, dactylitis, and spine symptoms. The tender and swollen joint counts are central to the measurement of the peripheral arthritis element of MSK disease activity. While several joint counts exist ${ }^{7}$, there are no existing recommendations about which joint count to use in RCT or LOS measuring peripheral arthritis in PsA, and none have moved through the instrument selection process described by OMERACT.

The goal of the PsA workshop at OMERACT was to seek endorsement of the 66/68 swollen and tender joint counts (SJC66/TJC68; Figure 2) as one of the instruments for the PsA COS. In this paper, we describe the instrument selection process as recommended by OMERACT, summarize the plenary presentation, and present the voting results and discussion points from the PsA workshop and breakout groups at the OMERACT 2018 meeting.

\section{METHODS AND RESULTS}

Patient engagement in the working group. One of the key tenets of the OMERACT process is involving patient research partners (PRP) in the process of developing core outcome sets. In the work presented in this paper, PRP have been involved in all aspects of the project: 3 PRP are part of the GRAPPA-OMERACT working group steering committee. They have reviewed and provided feedback on protocols, prereading materials, and presentations, and helped plan the workshop. Further, PRP from GRAPPA and OMERACT have participated in small groups and were involved in surveys and Web-based seminars.

Instrument selection process. Using the OMERACT Filter 2.1 Instrument Selection Process (Figure 3), an instrument is first assessed for "Truth: domain match" and "Feasibility," and if these 2 steps are met, the instrument may progress to the subsequent steps, "Truth 2: Numerical Sense" (i.e., construct validity) and "Discrimination" (measured by test-retest reliability, longitudinal construct validity, ability to distinguish between treatment and placebo groups in clinical trials, and thresholds of meaning $)^{8,9}$. To seek endorsement of an instrument, the working group assembles the evidence for the instrument, appraises it, and provides an overall assessment of the instrument using a Summary of Measurement Properties (SOMP) table. In the absence of evidence in the available literature, new studies may be performed by the working group to fill the evidence void. The working group makes a recommendation for endorsement and the attendees then vote on whether they agree with this recommendation. At OMERACT, the voting groups are split into PRP and others to ensure that the patient voice is adequately represented. At least $70 \%$ agreement among voting attendees at the session from both groups suggests consensus with the working group recommendation ${ }^{6}$. For a

Personal non-commercial use only. The Journal of Rheumatology Copyright $\subset$ (2019. All rights reserved. 


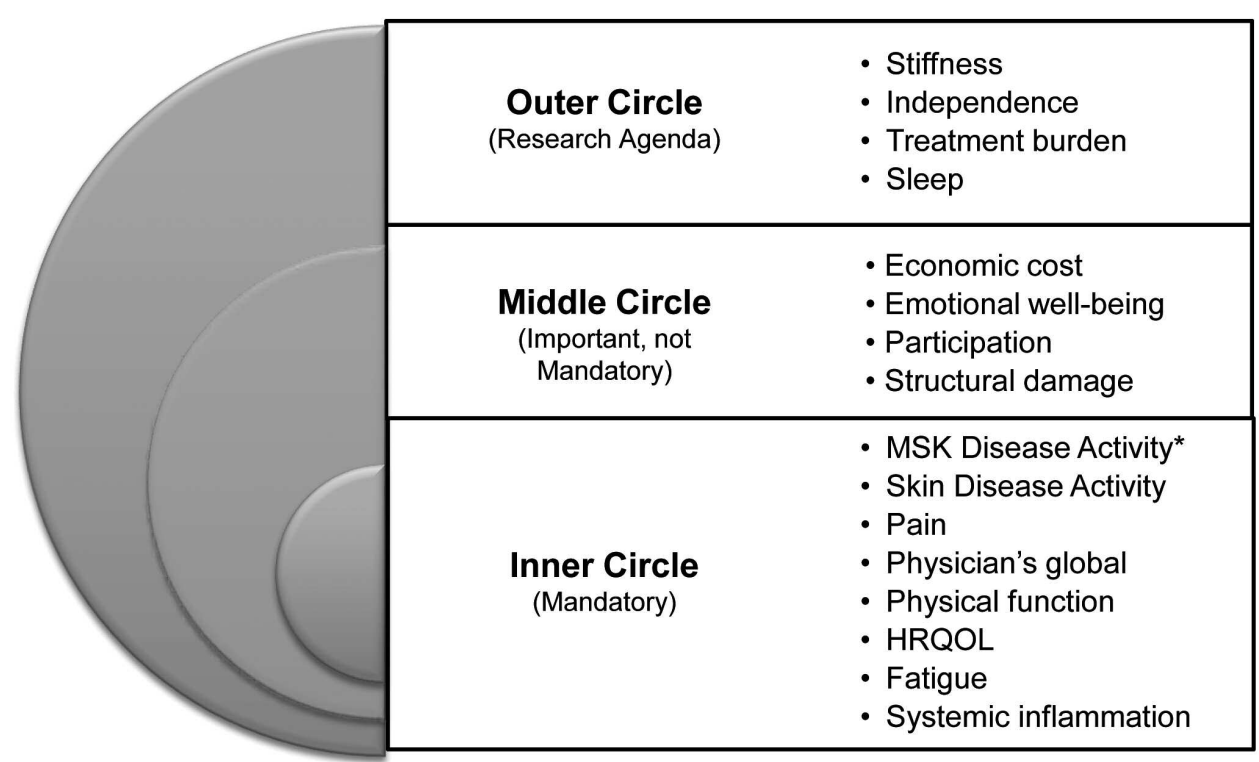

Figure 1. Updated 2016 psoriatic arthritis core domain set. Final set of core domains to be measured in randomized clinical trials and longitudinal observational studies, endorsed by OMERACT in $2016 . *$ MSK disease activity includes peripheral joints, enthesitis, dactylitis, and spine symptoms. OMERACT: Outcome Measures in Rheumatology; MSK: musculoskeletal; HRQOL: health-related quality of life.

\begin{tabular}{|c|c|c|}
\hline & Swollen joints & Tender joints \\
\hline Temporomandibular joint & ${ }^{(0-2)}$ & $\longrightarrow^{(0-2)}$ \\
\hline Sternoclavicular joints & ]$^{(0-2)}$ & $L^{(0-2)}$ \\
\hline Acromioclavicular joints & ${ }^{(0-2)}$ & ${ }^{(0-2)}$ \\
\hline Glenohumeral(s) & ${ }^{(0-2)}$ & ${ }^{(0-2)}$ \\
\hline Elbow(s) & ]$^{(0-2)}$ & $\Psi^{(0-2)}$ \\
\hline Wrist(s) & ${ }^{(0-2)}$ & $(0-2)$ \\
\hline Metacarpal phalangeal joints & ]$^{(0-10)}$ & $(0-10)$ \\
\hline $\begin{array}{l}\text { Finger Proximal } \\
\text { interphalangeal joints }\end{array}$ & $-^{(0-10)}$ & $-^{(0-10)}$ \\
\hline $\begin{array}{l}\text { Finger Distal } \\
\text { interphalangeal joints }\end{array}$ & $\underbrace{(0-8)}$ & $\underbrace{(0-8)}$ \\
\hline Hip(s) & NA & ${ }^{(0-2)}$ \\
\hline Knee(s) & $(0-2)$ & $(0-2)$ \\
\hline Ankle(s) & ]$^{(0-2)}$ & ${ }^{(0-2)}$ \\
\hline Tarsus/Midfoot(feet) & ${ }^{(0-2)}$ & ${ }^{(0-2)}$ \\
\hline Metatarsal phalangeal joints & ${ }^{(0-10)}$ & ${ }^{(0-10)}$ \\
\hline Toe PIP(s) & ]$^{(0-10)}$ & ${ }^{(0-10)}$ \\
\hline Total joint counts & ${ }_{(0-66)}$ & ${ }^{(0-68)}$ \\
\hline
\end{tabular}
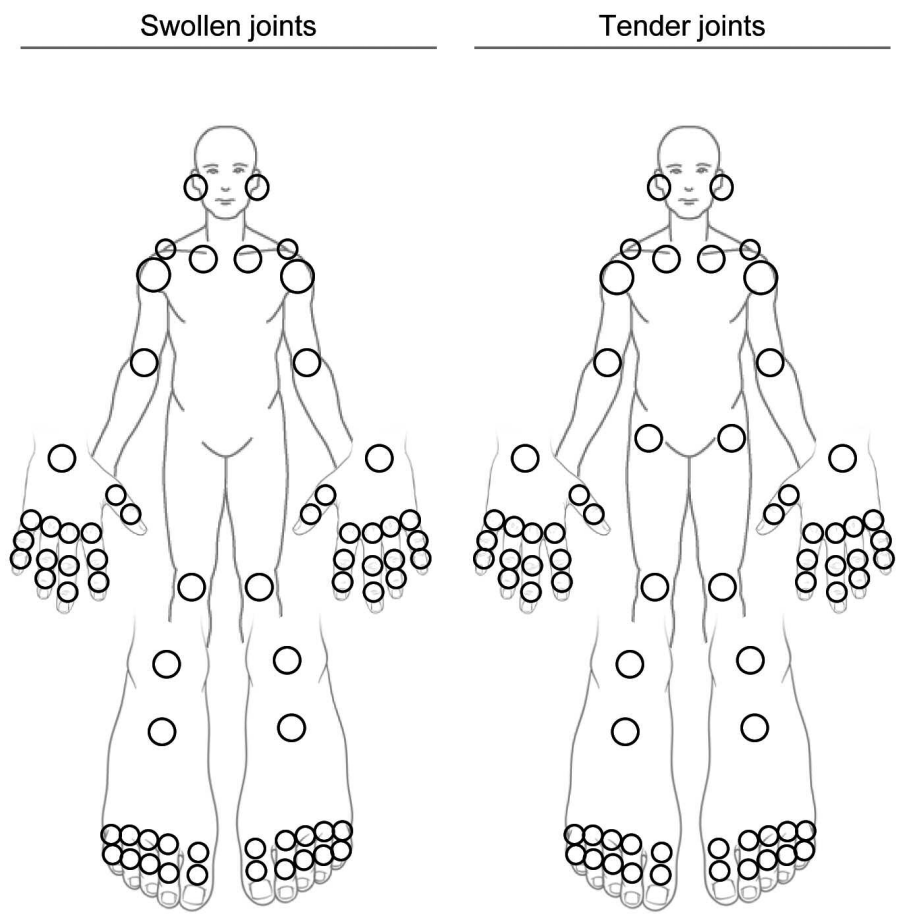

Figure 2. The SJC66/TJC68 joint count. The 66 swollen and 68 tender joints are assessed (the hips are not assessed for swelling). The joint count is scored as a sum of the tender joints and a sum of the swollen joints. SJC66: swollen joint count in 66 joints; TJC68: tender joint count in 66 joints.

more in-depth review of the instrument selection process, see the OMERACT Handbook ${ }^{8}$. The research protocol was reviewed and approved by the Institutional Review Board
(IRB) of the University of Pennsylvania (IRB PROTOCOL\#: 829776) for the PRP surveys and Webinars, while the rest of the project components were deemed exempt from IRB review. 


\section{How to choose an instrument}

\section{the OMERACT way}
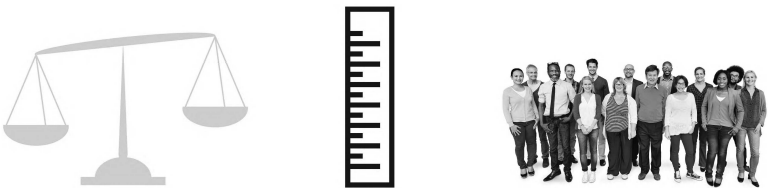

understand what you are trying

to measure

review domain

definition and

context of

measurement

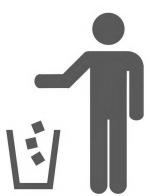

narrow the field

set aside any

red-flagged

instruments.

short list the

instruments.
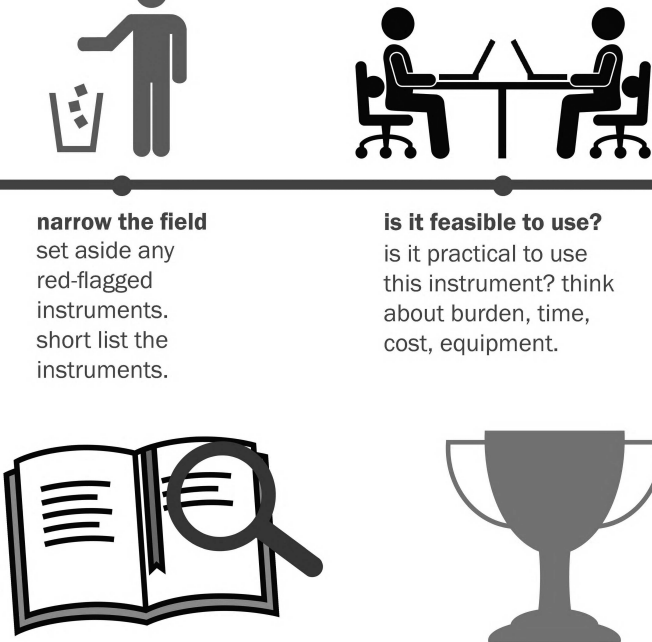

is it feasible to use?

is it practical to use

this instrument? think

about burden, time

cost, equipment.

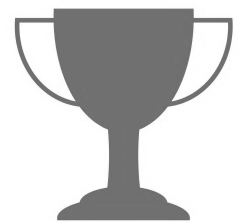

gather the evidence

find, appraise and synthesize

the measurement property

evidence. look for consistent

findings of good performance

from good quality studies. identify the winners

identify the winners:

those that have passed

the filter 2.1. discuss work

with Technical Advisory

Group

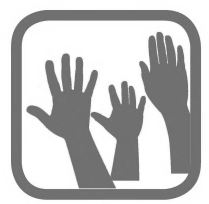

O

bring it

to a vote

brought to you by: Technical Advisory Group of

OMERACT Filter 2.0

OMERACT Filter 2.0
Figure 3. OMERACT instrument selection process. Selecting an instrument the OMERACT Way means proceeding through the steps shown in the figure: first defining the domain to be measured, identifying candidate instruments, assessing whether the instruments match the domain and feasibility, narrowing the list (removing instruments that do not match the domain), gathering evidence about measurement properties in a systematic literature search and data analysis, identifying the winners, and taking to OMERACT for endorsement. This figure was designed by Dorcas Beaton, OMERACT Handbook; used with permission. OMERACT: Outcome Measures in Rheumatology. 
Trial participants in the original trials completed informed consent prior to participation. Patients who did not give consent for their data to be used for other studies were excluded from the additional trial analyses.

Evaluation of joint counts using the OMERACT process. A systematic literature search (SLR) was first performed to identify instruments that had been used to measure MSK disease activity, which includes peripheral joint activity, enthesitis, dactylitis, and spine symptoms in PsA, and to assess their measurement properties ${ }^{10,11}$. In our report, we focused on the evidence evaluating the SJC28/TJC28, SJC66/TJC68, and SJC76/TJC78. We addressed domain match and feasibility at the GRAPPA meeting in 2017 (Amsterdam, the Netherlands), as well as with the working group and PRP (described in more detail below). We assessed the measurement properties of the joint counts in the literature (and applied the OMERACT Good Methods Checklist to assess data quality) and analyzed measurement properties in clinical trial and LOS datasets (obtained from companies and principal investigators). The working group requested data from phase III trials published between 2010-2017 and was included from 7 phase III RCT, The Tight Control of Inflammation in Psoriatic Arthritis trial, and 1 LOS, the Psoriatic Arthritis Research Consortium. A priori, a standardized protocol was designed to address content validity, construct validity, responsiveness, and discrimination.

We used these data to complete the SOMP table and presented this to the working group for a final recommendation. The results were then presented at the OMERACT meeting in Terrigal, Australia.

The PsA OMERACT Core Set Workshop at the GRAPPA 2017 meeting: domain match and feasibility of the joint counts as discussed by clinicians and other stakeholders. Domain match and feasibility for the SJC66/TJC68 were addressed at GRAPPA 2017 in a breakout group discussion, and following the meeting, among working group members using a Web-based survey. During the GRAPPA meeting, content validity and feasibility were addressed within a small group with clinicians, 2 patients, and a patient advocate; the voting sheets were completed by 22 people $^{12}$. There was consensus $(20 / 22,91 \%)$ among the group that the SJC66/TJC68 was a match for the MSK disease activity/peripheral arthritis domain and that there was adequacy of content and no redundancies. Regarding feasibility, all the voters agreed that the SJC66/TJC68 was feasible.

Eighteen working group members completed a followup online survey. This survey documented the reasons for selecting SJC66/TJC68 count over the comparators (28 and $76 / 78$ joint counts). The 28 -joint count is a core measure for rheumatoid arthritis (RA) and is frequently performed in clinical practice. The 76/78 joint count is performed in some trials. Other joint counts beyond the $28,66 / 68$, and $76 / 78$ (i.e., 32, 44, Ritchie index) were not sufficiently used in RCT or LOS to merit inclusion ${ }^{7}$.
Concerns have been raised about these joint counts in PsA: the 28-joint count does not include the joints of the feet, and those joints are frequently affected in PsA; this concern was raised by both PRP and clinicians. The 76/78 joint count includes the carpometacarpal (CMC) joints, typically involved in osteoarthritis (OA), and thus tenderness in this joint is difficult to attribute to PsA, and it separately includes the toe proximal and distal interphalangeal joints; these joints are difficult to decipher individually on examination, decreasing feasibility.

The 28-joint count did not meet domain match (does not cover key joints) and the 76/78-joint count had lower feasibility (difficult to distinguish between toe joints) and reduced domain match (CMC joint more often an OA joint) compared to the SJC66/TJC68. Given the results of the above discussions and surveys with all stakeholders, the working group decided to move forward only the SJC66/TJC68 through the OMERACT Filter (Figure 4).

Domain match and feasibility of the joint counts: PRP. To assess domain match and feasibility from the PRP perspective, a Web-based survey was designed with an embedded video of a clinician (AO) performing the SJC66/TJC68. Respondents were asked to note whether they agreed that the SJC66/TJC68 measured their perception of "peripheral arthritis disease activity" and whether it was feasible to complete within RCT or LOS visits. PRP representatives of GRAPPA and OMERACT were invited to participate in the survey, and 14 responded. Among those who responded, 9 voted green, 3 voted amber, and 1 voted white. For feasibility, 13 voted green and 1 voted white. After completion of the survey, 2 Web-based seminars were held with the participating PRP to discuss the results. Points of confusion with the domain were that several patients did not endorse for "domain match" because the SJC66/TJC68 did not include the entheses or the spine. AO reminded the group that enthesitis and spine symptoms are assessed using separate measures, and this explanation was satisfactory to those who voted "no" (although the group did not re-vote because the vote was mainly used to start the discussion). Some patients advocated for inclusion of the CMC joint as a common source of pain. PRP also noted that the feet and ankles are essential for inclusion in assessing peripheral arthritis in PsA.

Regarding feasibility, all patients felt that the SJC66/TJC68 is feasible. The only concern raised was that when patients are in a lot of pain, getting shoes on and off is uncomfortable and can decrease feasibility. Additionally, the patient needs sufficient time to respond during examination (i.e., if the SJC66/TJC68 is performed too quickly, there will not be sufficient opportunity to say "yes" to a tender joint). It was also noted by several PRP that for the joint count to be a valid assessment of peripheral arthritis, particularly tenderness, there needs to be communication between the physician and patient. There was discussion about the fact

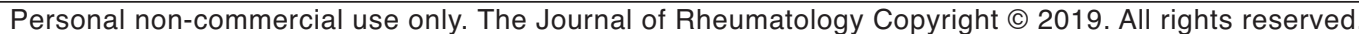




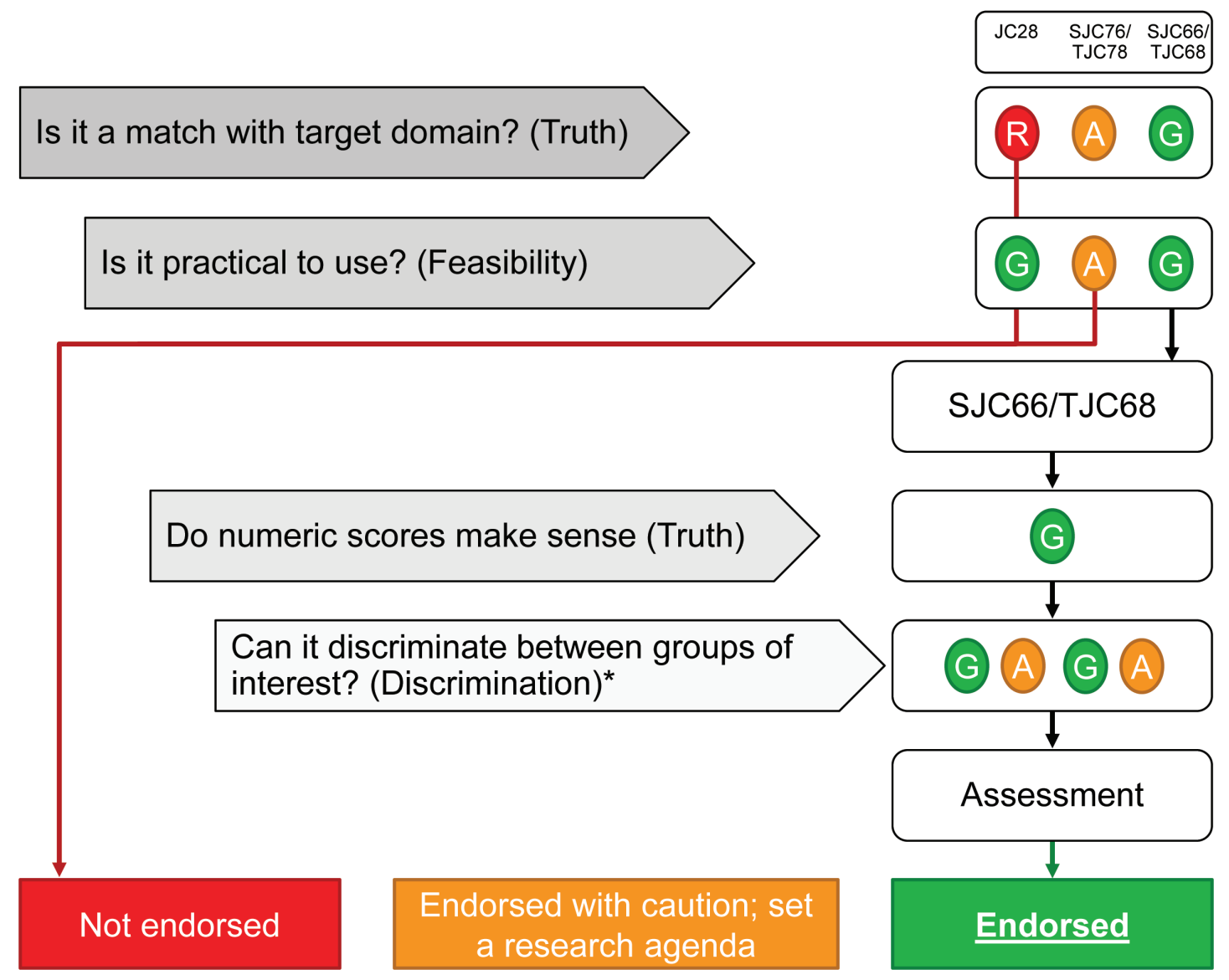

Figure 4. The OMERACT Filter 2.1 instrument selection process for the 66/68 swollen and tender joint counts (SJC66/TJC68). *Discrimination includes longitudinal construct validity, clinical trial discrimination (green circles), test-retest reliability and thresholds of meaning (amber circles). JC28: 28/28 swollen and tender joint counts; SJC76: 76 swollen joint counts; TJC78: 78 tender joint counts.

that the joint examination may miss a joint that was active within the past week but is not active that day. Finally, patients said that there was no clear meaning for "tender" and that communication from the physician prior to the joint count is assessed is needed.

Numerical sense (construct validity) and discrimination. We addressed numerical sense and discrimination through an SLR and analysis of RCT datasets. In the SLR, 1921 unique references regarding the 4 components of the MSK disease activity domain were identified, 159 were eligible for full-text article assessment, and 87 of these were excluded in this phase. Fifty-nine of the 72 remaining were excluded because they involved other components of the MSK disease domain (e.g., dactylitis) that were not pertinent for this report or because of a lack of enough data regarding the SJC66 and TJC68. Thirteen SJC66/TJC68 unique references were included in the good methods analysis. The good methods checklist is applied at the level of the instrument and measurement property tested rather than the level of the study; in our case, no study had some red and other evidence that was amber or green. Three studies had all their evidence as red and therefore were excluded, leaving 10 studies for inclusion (Figure 5).

The list of articles and summary of findings were included in Table $1^{13-27}$. The results suggest that SJC66 and TJC68 have construct validity. TJC68 has adequate interrater reliability while SJC66 does not have adequate interrater reliability $(\mathrm{ICC}<0.75)^{28}$. Regarding responsiveness and discrimination, SJC66 and TJC68 change over time in response to treatment (placebo did change as well but less) and the change in SJC66 and TJC68 can distinguish between patients receiving an effective therapy compared to placebo. We similarly addressed measurement properties including responsiveness and discrimination in RCT datasets (manuscript in progress, data presented at the OMERACT meeting). Standardized response means ranged from -0.9 to -0.5 for the SJC66 and -0.9 to -0.4 for the TJC68, thus mostly in the moderate effect range. Standardized mean difference (treatment compared to placebo) range from -0.7 to -0.2 for the SJC66 and -0.6 to -0.2 for the TJC68.

Personal non-commercial use only. The Journal of Rheumatology Copyright (c) 2019. All rights reserved. 


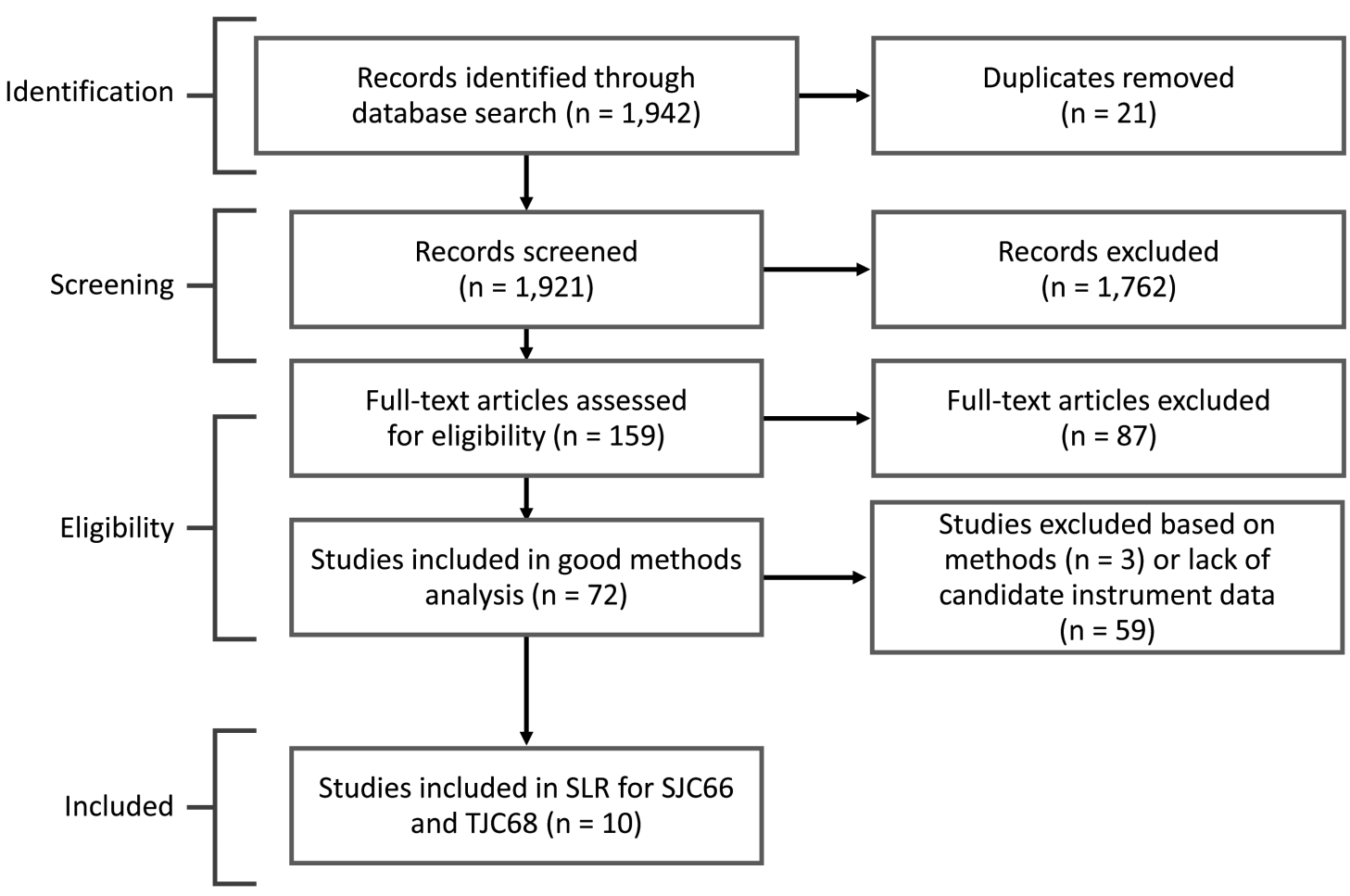

Figure 5. PRISMA diagram. We conducted a systematic literature review for all 4 components of "MSK Disease Activity" that includes peripheral arthritis, enthesitis, dactylitis, and spine symptoms. In the current report, we focused on the TJC68 and SJC66 and thus many manuscripts went through the good methods checklist but only 10 papers included measurement properties that met these criteria and applied to the joint counts. In our case, no study had some red and other evidence that was amber or green. Three studies had all their evidence as red and therefore were excluded. PRISMA: Preferred Reporting Items for Systematic reviews and Meta-Analyses; MSK: musculoskeletal; SLR: systematic literature review; SJC66: 66 swollen joint count; TJC68: 68 tender joint count.

The working group concluded that the SJC66/TJC68 meets the OMERACT criteria for domain match, feasibility, truth, and discrimination. The instruments' shortfalls are relatively low interrater reliability for the SJC only and a lack of studies addressing intrarater reliability of the TJC/SJC in PsA (Table 1).

OMERACT 2018 PsA Workshop: plenary presentation and breakout group discussions. In the plenary presentation, we presented the evidence that addressed each of the 4 steps of the OMERACT Filter 2.1 Instrument Selection Process for SJC66/TJC68. Data from these studies were summarized in the SOMP table (Table 1).

After the plenary presentation, 8 breakout groups were asked to discuss the 4 measurement properties (content validity/domain match, feasibility, construct validity, and discrimination) and vote on agreement with the working group's assessment of green (good to go), amber (some concerns raised), or red (not endorsed). Breakout groups were facilitated by 1 OMERACT-trained facilitator and 1 reporter; reporters were part of the working group or experienced researchers. During breakout groups, the participants had the option to raise concerns regarding the working group assessment of green. Overall, most participants agreed with our assignment of green for content validity/domain match, feasibility, and construct validity. Feasibility concerns came up for some groups in that the SJC66/TJC68 takes longer than the reduced joint counts, but overall, the majority felt that the SJC66/TJC68 is feasible in the setting of an RCT or LOS. In some groups, concerns were raised about discrimination, mainly centered around the insufficient data for test-retest reliability and thresholds of meaning (both with only 1 unpublished study available in PsA). Additionally, the concern about the relatively low interrater reliability of the SJC was raised. This was countered by the argument that in most RCT, the assessor is the same throughout the study and test-retest reliability, or intrarater reliability, in a single unpublished study was found to be quite high (ICC 0.8-0.9; Tillett, et al, unpublished). Further, clinicians are generally asked to undergo training prior to trial participation to increase interrater reliability ${ }^{13}$. Reasons for endorsement of the SJC66/TJC68 that were raised included sending a clear message that this is the preferred joint count based upon evidence to assist in standardizing joint counts among RCT.

A broader discussion was raised in the small groups regarding the meaning of full endorsement of an instrument (green) or provisional endorsement (amber). Some wondered 
whether a green instrument would then become mandatory, similar to the inner circle of a core domain set. However, in the PsA workshop, green was used to denote the sufficient measurement properties to confidently say that the instrument is good, and amber was used to indicate that although this is a in all other domains collectively being excellent; the studies evaluating test-retest reliability and thresholds of meaning being sufficient; and further research on these domains, though supportive, is not critical to further inform the preferred use of the SJC66/TJC68 over other joint counts.

Table 1. Summary of measurement properties table for TJC68/SJC66.

\begin{tabular}{|c|c|c|c|c|c|c|c|c|}
\hline \multirow[t]{2}{*}{ Author/Year } & \multirow{2}{*}{$\begin{array}{c}\text { Truth } \\
\text { Domain } \\
\text { Match* }\end{array}$} & \multirow[t]{2}{*}{ Feasibility* } & \multirow{2}{*}{\begin{tabular}{|c|} 
Truth \\
Construct \\
Validity
\end{tabular}} & \multicolumn{5}{|c|}{ Discrimination } \\
\hline & & & & $\begin{array}{l}\text { Test-Retest } \\
\text { Reliability }\end{array}$ & $\begin{array}{l}\text { Interrater } \\
\text { Reliability }\end{array}$ & $\begin{array}{c}\text { Longitudinal } \\
\text { Construct Validity }\end{array}$ & \begin{tabular}{|l} 
Clinical Trial \\
Discrimination
\end{tabular} & $\begin{array}{c}\text { Thresholds of } \\
\text { Meaning }\end{array}$ \\
\hline Aalbers, et $a l^{17}$ & & & & & & + & \pm & \\
\hline Chandran, et $a l^{18}$ & & & & & $+(\mathrm{TJC}) /-(\mathrm{SJC})$ & & & \\
\hline Fransen, et al ${ }^{19}$ & & & & & & + & + & \\
\hline Gladman, et $a l^{20}$ & & & & & $+(\mathrm{TJC}) /-(\mathrm{SJC})$ & & & \\
\hline Gladman, et $a l^{21}$ & & & & & $+(\mathrm{TJC}) /-(\mathrm{SJC})$ & & & \\
\hline Husic, et $a l^{22}$ & & & + & & & & & \\
\hline Leung, et $a l^{23}$ & & & + & & & & & \\
\hline Lubrano, et al ${ }^{24}$ & & & + & & & & & \\
\hline Tillet, et $a l^{25}$ & & & & & - & & & \\
\hline Salvarani, et al ${ }^{13}$ & & + & & & $+(\mathrm{TJC}) /-(\mathrm{SJC})$ & & & \\
\hline $\begin{array}{c}\text { Duarte-García, et al (data } \\
\text { analysis; unpublished) }\end{array}$ & & & \pm & & & + & + & \\
\hline $\begin{array}{l}\text { Tillett, et al } \\
\text { (unpublished) }\end{array}$ & & & & + & & & & + \\
\hline $\begin{array}{l}\text { GRAPPA and Working Group } \\
\text { Surveys }\end{array}$ & + & + & & & & & & \\
\hline Patient input & + & + & & & & & & \\
\hline Thompson, et al 26 & & & & + & & & & \\
\hline Deandrade, et $a l^{27}$ & & & & + & & & & \\
\hline $\begin{array}{l}\text { Total available studies for } \\
\text { each property }\end{array}$ & 2 & 3 & 4 & 3 & 5 & 3 & 3 & 1 \\
\hline $\begin{array}{l}\text { Total studies available } \\
\text { for synthesis }\end{array}$ & 2 & 3 & 4 & 3 & 5 & 3 & 3 & 1 \\
\hline Rating & Green & Green & Green & Amber & $\begin{array}{l}\text { Green for TJC } \\
\text { Amber for SJC }\end{array}$ & Green & Green & Amber \\
\hline $\begin{array}{l}\text { Overall rating for instrument } \\
\text { across properties }\end{array}$ & & & en: Wor & roup Is $\mathrm{Re}$ & ommending Endo & sement & & \\
\hline
\end{tabular}

The color indicates the good methods checklist/recommendation; the \pm sign indicates the adequacy of the data in support of the instrument. For test-retest reliability, we have provided primary data (1 study). After identifying the gap in test-retest reliability in PsA, a hand search for test-retest reliability in RA was conducted to provide additional evidence and context about this property in the assessment of peripheral arthritis (2 studies). Some studies were excluded because the measurement properties tested did not meet the good methods checklist: Gladman, et al ${ }^{14}$ (test-retest reliability, necessary summary statistics not provided); Englbrecht, et al ${ }^{15}$ (construct validity, only tested correlation among subsets of TJC68 and SJC66); Schoels, et al ${ }^{16}$ (construct validity and longitudinal construct validity, did not test associations of interest between joint counts and other individual measures). TJC68: 68 tender joint count; SJC66: 66 swollen joint count; PsA: psoriatic arthritis; RA: rheumatoid arthritis; GRAPPA: Group for Research and Assessment of Psoriasis and Psoriatic Arthritis.

good instrument that could be used, further research is still required on its measurement properties. It is possible that multiple instruments for the same domain will pass through the filter at a green level, thus requiring a subsequent consensus process to identify the best instrument. Additional discussion then turned to define "good enough." We assigned an amber to test-retest reliability and thresholds of meaning because of only 1 unpublished study for each. The OMERACT Handbook suggests that the instrument should then be amber. However, the working group felt that the instrument (SJC66/TJC68) should be endorsed as "green" given the data
Vote for the 66/68 joint counts. Following report back from the groups and discussion, a vote was held for the endorsement of the instrument. Among PRP, 100\% (of 14 patient votes in total) voted for a green endorsement. Among all other stakeholders, $88 \%$ ( 84 of 96 votes in total) voted for a green endorsement.

\section{DISCUSSION}

Through the years, the lack of standardization of the instruments to measure peripheral arthritis in PsA has resulted in the use of different instruments in RCT and LOS.

\section{Personal non-commercial use only. The Journal of Rheumatology Copyright $\odot$ 2019. All rights reserved.}


After a careful assessment by PRP, clinicians, methodologists, representatives of the pharmaceutical industry, and other stakeholders, and in accordance with the OMERACT Filter 2.1, the evidence supporting the measurement properties of the SJC66/TJC68 was assessed and resulted in full endorsement (green) by OMERACT as an instrument to measure MSK disease activity/peripheral arthritis in PsA. The SJC66/TJC68 is the first green instrument to enter the PsA COS.

The MSK disease activity domain includes the heterogeneous disease manifestations of PsA: enthesitis, dactylitis, spondylitis/axial arthritis, and peripheral arthritis. An ongoing program will assess and eventually seek endorsement of the optimal instruments that measure the other components of the MSK disease activity domain. While the joint count is the first to go through the filter for this domain, others will be added in the future as the additional work streams proceed through additional systematic literature reviews and consensus processes. In the meantime, PRP, regulatory agencies, investigators developing protocols for RCT and LOS, and other stakeholders can be confident with the SJC66/TJC68, and adoption of the SJC66/TJC68 will be monitored in published RCT and LOS.

\section{ACKNOWLEDGMENT}

We thank Kathleen Bush and Christina Burgese for administrative support. We also thank Janssen Scientific Affairs LLC for its assistance in identifying access to trial data through the YODA (Yale Open Data Access) Project. We would like to thank UCB, Novartis, and Pfizer for their scientific partnership by analyzing their clinical data of the certolizumab RAPID-PsA, secukinumab FUTURE I \& II, and tofacitinib PsA OPAL studies, respectively, to support the OMERACT-GRAPPA working group.

\section{REFERENCES}

1. Orbai AM, de Wit M, Mease P, Shea JA, Gossec L, Leung YY, et al. International patient and physician consensus on a psoriatic arthritis core outcome set for clinical trials. Ann Rheum Dis 2017;76: 673-80.

2. Orbai AM, de Wit M, Mease PJ, Callis Duffin K, Elmamoun M, Tillett W, et al. Updating the psoriatic arthritis (PsA) core domain set: a report from the PsA workshop at OMERACT 2016. J Rheumatol 2017;44:1522-8.

3. Ogdie A, de Wit M, Callis Duffin K, Campbell W, Chau J, Coates LC, et al. Defining outcome measures for psoriatic arthritis: a report from the GRAPPA-OMERACT working group. J Rheumatol 2017;44:697-700.

4. Tillett W, Orbai AM, Ogdie A, Leung YY, Strand V, Gladman DD, et al; GRAPPA OMERACT Psoriatic Arthritis working group. GRAPPA-OMERACT initiative to standardise outcomes in psoriatic arthritis clinical trials and longitudinal observational studies. Ann Rheum Dis 2018;77:e23.

5. Prinsen CA, Vohra S, Rose MR, Boers M, Tugwell P, Clarke M, et al. How to select outcome measurement instruments for outcomes included in a "core outcome set" - a practical guideline. Trials 2016; 17:449.

6. Beaton DE, Maxwell LJ, Shea BJ, Wells GA, Boers M, Grosskleg S, et al. Instrument selection using the OMERACT filter 2.1: The OMERACT methodology. J Rheumatol 2019;46:1028-35.

7. Sokka T, Pincus T. Quantitative joint assessment in rheumatoid arthritis. Clin Exp Rheumatol 2005;23 Suppl 39:S58.
8. Boers M, Kirwan JR, Tugwell P, Beaton D, Bingham CO III, Conaghan PG, et al. The OMERACT Handbook. [Internet. Accessed May 17, 2017.] Available from: https://omeract.org/resources

9. Boers M, Kirwan JR, Wells G, Beaton D, Gossec L, d'Agostino MA, et al. Developing core outcome measurement sets for clinical trials: OMERACT filter 2.0. J Clin Epidemiol 2014;67:745-53.

10. Duarte-Garcia A, Eder L, Stephens-Shields A, Goel N, de Witt M, Gladman D, et al. Construct validity of the swollen and tender joint counts for the measurement of MSK disease activity in psoriatic arthritis [abstract]. Arthritis Rheumatol 2018;70 Suppl 10:641.

11. Duarte-Garcia A, Eder L, Goel N, Christensen R, de Wit M, FitzGerald O, et al. The 66/68 joint count for the measurement of MSK disease activity/peripheral joint activity in PSA: A

GRAPPA-OMERACT working group initiative [abstract]. Arthritis Rheumatol 2018;70 Suppl 10:1606.

12. Holland R, Tillett W, Ogdie A, Leung YY, Gladman DD, Callis Duffin K, et al. Content and face validity and feasibility of 5 candidate instruments for psoriatic arthritis randomized controlled trials: the PsA OMERACT core set workshop at the GRAPPA 2017 annual meeting. J Rheumatol Suppl. 2018 Jun;94:17-25.

13. Salvarani C, Girolomoni G, Di Lernia V, Gisondi P, Tripepi G, Egan $\mathrm{CG}$, et al; DREAM study group. Impact of training on concordance among rheumatologists and dermatologists in the assessment of patients with psoriasis and psoriatic arthritis. Semin Arthritis Rheum 2016;46:305-11

14. Gladman DD, Farewell V, Buskila D, Goodman R, Hamilton L, Langevitz P, et al. Reliability of measurements of active and damaged joints in psoriatic arthritis. J Rheumatol 1990;17:62-4.

15. Englbrecht M, Wang Y, Ronneberger M, Manger B, Vastesaeger N, Veale DJ, et al. Measuring joint involvement in polyarticular psoriatic arthritis: an introduction of alternatives. Arthritis Care Res 2010;62:977-83.

16. Schoels M, Aletaha D, Funovits J, Kavanaugh A, Baker D, Smolen JS. Application of the DAREA/DAPSA score for assessment of disease activity in psoriatic arthritis. Ann Rheum Dis 2010; 69:1441-7.

17. Aalbers CJ, Gerlag DM, Vervoordeldonk MJ, Tak PP, Landewe RB. Single-joint assessment for the evaluation of intraarticular treatment: responsiveness and discrimination of the composite change index. J Rheumatol 2015;42:1672-6.

18. Chandran V, Gottlieb A, Cook RJ, Duffin KC, Garg A, Helliwell P, et al. International multicenter psoriasis and psoriatic arthritis reliability trial for the assessment of skin, joints, nails, and dactylitis. Arthritis Rheum 2009;61:1235-42.

19. Fransen J, Antoni C, Mease PJ, Uter W, Kavanaugh A, Kalden JR, et al. Performance of response criteria for assessing peripheral arthritis in patients with psoriatic arthritis: analysis of data from randomised controlled trials of two tumour necrosis factor inhibitors. Ann Rheum Dis 2006;65:1373-8.

20. Gladman DD, Helliwell P, Mease PJ, Nash P, Ritchlin C, Taylor W. Assessment of patients with psoriatic arthritis: a review of currently available measures. Arthritis Rheum 2004;50:24-35.

21. Gladman DD, Inman RD, Cook RJ, van der Heijde D, Landewe RB, Braun J, et al. International spondyloarthritis interobserver reliability exercise - the INSPIRE study: I. Assessment of spinal measures. J Rheumatol 2007;34:1733-9.

22. Husic R, Gretler J, Felber A, Graninger WB, Duftner C, Hermann J, et al. Disparity between ultrasound and clinical findings in psoriatic arthritis. Ann Rheum Dis 2014;73:1529-36.

23. Leung YY, Ho KW, Zhu TY, Tam LS, Kun EW, Li EK. Construct validity of the modified numeric rating scale of patient global assessment in psoriatic arthritis. J Rheumatol 2012;39:844-8.

24. Lubrano E, Perrotta FM, Parsons WJ, Marchesoni A. Patient's global assessment as an outcome measure for psoriatic arthritis in

Personal non-commercial use only. The Journal of Rheumatology Copyright $\odot$ (2019. All rights reserved 
clinical practice: a surrogate for measuring low disease activity? J Rheumatol 2015;42:2332-8.

25. Tillett W, Shaddick G, Korendowych E, de Vries CS, McHugh N. Joint count reliability in psoriatic arthritis observational trials - an unreported problem. Rheumatology 2012;51:1333-4.

26. Thompson PW, Hart LE, Goldsmith CH, Spector TD, Bell MJ, Ramsden MF. Comparison of four articular indices for use in clinical trials in rheumatoid arthritis: patient, order and observer variation. J Rheumatol 1991;18:661-5.
27. Deandrade JR, Casagrande PA. A seven-day variability study of 499 patients with peripheral rheumatoid arthritis. Arthritis Rheum 1965;8:302-34.

28. Streiner DL, Norman GR, Cairney J. Health measurement scales: a practical guide to their development and use. New York: Oxford University Press, USA; 2015. 